Short communication

\title{
Detection of Mastitis in Goat Milk using qRTPCR
}

Amber L. Martin, Ashley Newton, Yonathan Tilahun*, and Steve Zeng

School of Agriculture and Applied Sciences, Langston University, Langston, OK 73050, US

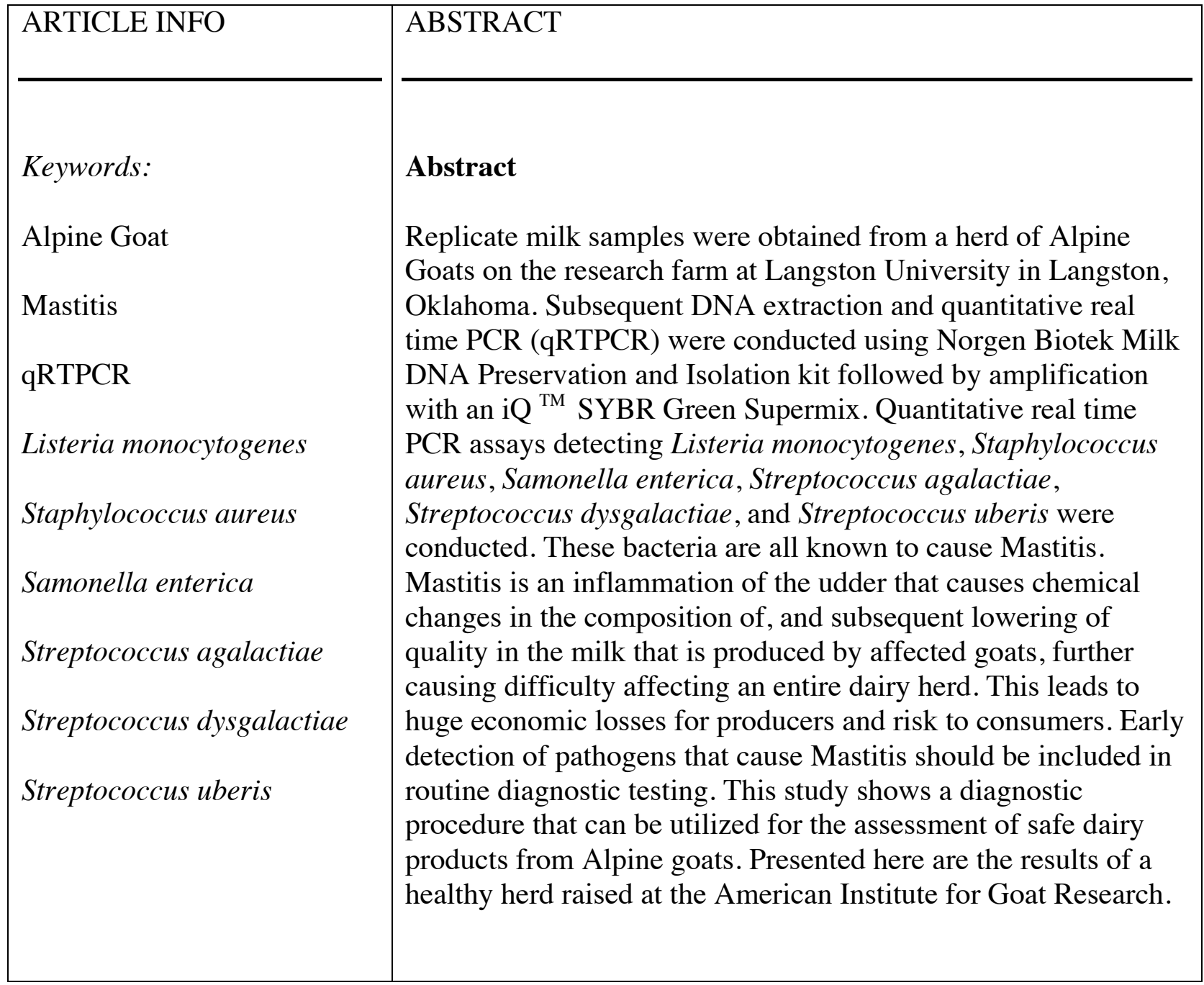

* Corresponding author at: American Institute for Goat Research at Langston University, Langston, OK 73034, US. E-mail address: ytilahun@langston.edu (Y. Tilahun) 


\section{Introduction}

Mastitis is an inflammation of the udder. This inflammation causes chemical changes in, and a lowering in quality of, milk produced by affected goats. The lowered quality subsequently affects production of additional commodities, like cheese. Mastitis is most commonly a development of infection by the following bacterium: Listeria monocytogenes, Staphylococcus aureus, Samonella enterica, Streptococcus agalactiae, Streptococcus dysgalactiae, and Streptococcus uberis. Mastitis can rapidly spread throughout a herd prior to the presentation of symptoms. In parlors using milk machines, bacterium on one udder quickly transfers from one goat to another. Once the goats present symptoms, most or all of the herd may be affected. This disease is costly in two ways: Veterinary costs of treating the goats; Loss in product and commodity as a result of infection/low quality milk.

Early detection of pathogens that cause mastitis will reduce the likelihood of mastitis outbreaks. Pathogens that cause mastitis could be detected early by routine diagnostic testing. Our study aims to identify a diagnostic procedure for utilization in assessment of safe dairy products from dairy goats. Presented here are the results of a healthy herd raised at the American Institute for Goat Research.

2. Materials and methods

Replicate milk samples were obtained from the herd of Alpine Goats on the research farm at Langston University in Langston, OK. DNA was extracted with a Norgen Biotek Milk DNA Preservation and Isolation Kit. DNA (Norgen Biotek (3430 Schmon Parkway Thorold, ON Canada, L2V4Y6) was amplified using an iQ ${ }^{\mathrm{TM}}$ SYBR Green Supermix. qRTPCR assays were conducted to detect: Listeria monocytogenes, Staphylococcus aureus, Samonella enterica, Streptococcus agalactiae, Streptococcus dysgalactiae, Streptococcus uberis. All primers for Mastitis causing bacteria were synthesized and obtained from Norgen Biotek.

* Corresponding author at: American Institute for Goat Research at Langston University, Langston, OK 73034, US. E-mail address: ytilahun@langston.edu (Y. Tilahun) 


\section{Results}

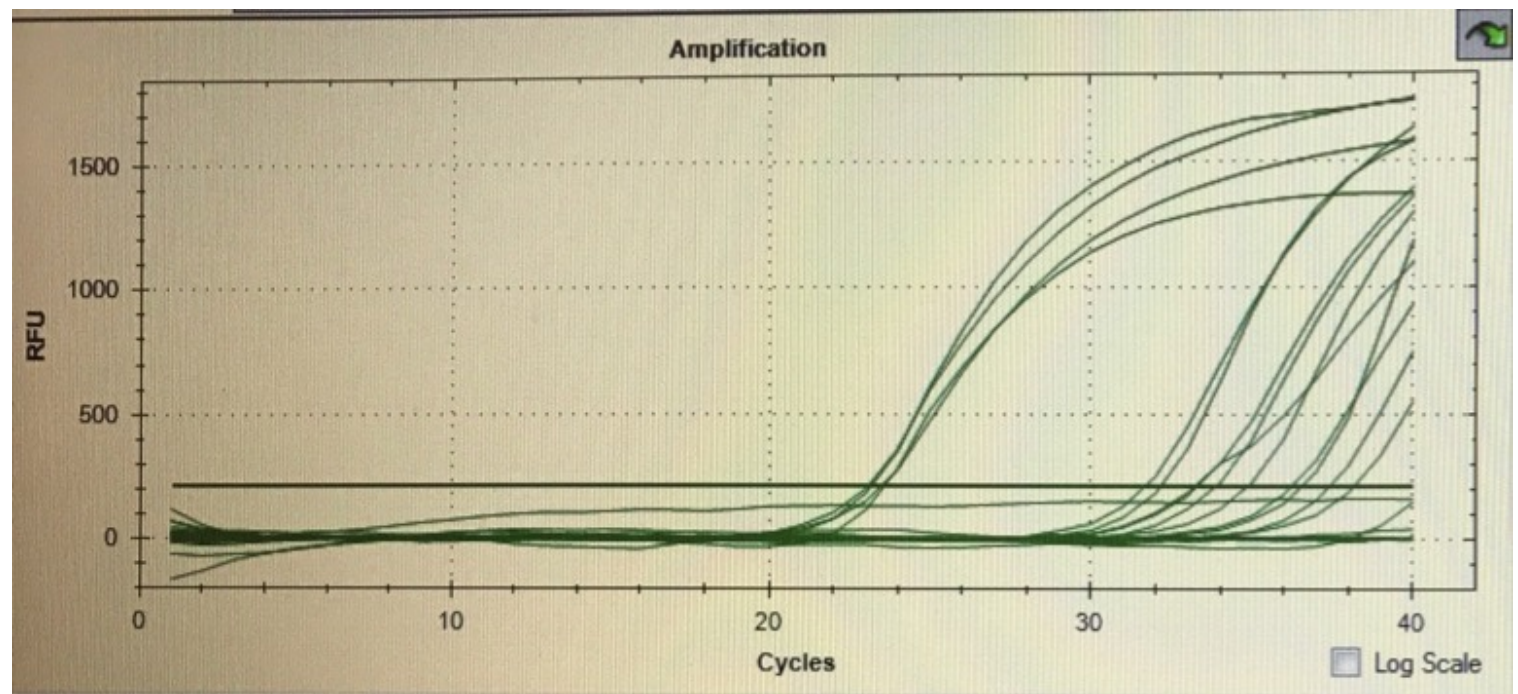

Fig. 1. qRTPCR analysis revealing Negative Controls were not detected, that the negative control samples did not begin amplification until following 30 cycles. Amplification signal in negative control samples prepared with all reaction components except the DNA template, referred to as a "no-template" or "primer-only" control.

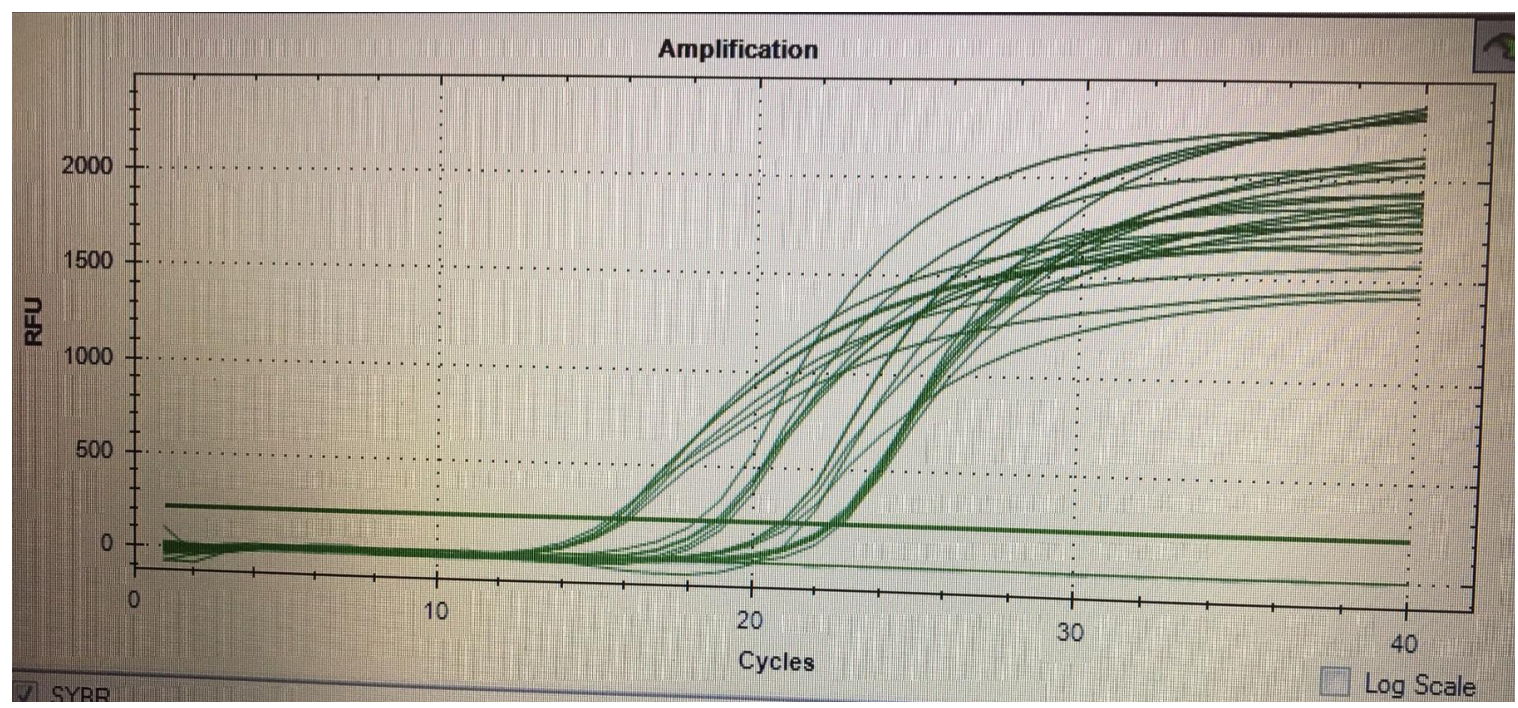

Fig. 2. qRTPCR analysis revealed samples with 18 S Primers all amplified well. All began amplifying with this positive control in $<20$ cycles.

* Corresponding author at: American Institute for Goat Research at Langston University, Langston, OK 73034, US. E-mail address: ytilahun@langston.edu (Y. Tilahun) 


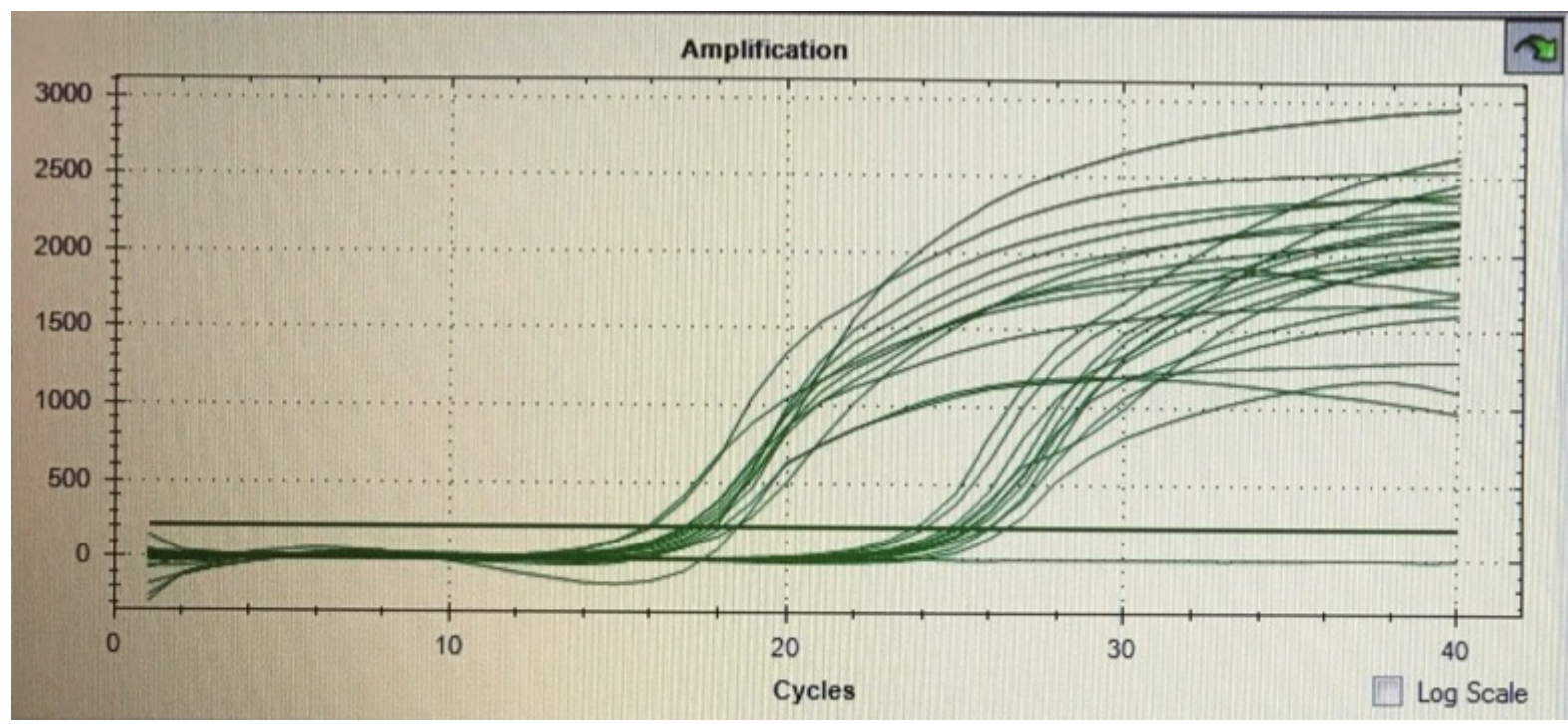

Fig. 3. qRTPCR analysis revealed Positive Controls (Bacterial DNA): Bacterial Primers combination showed success amplification.

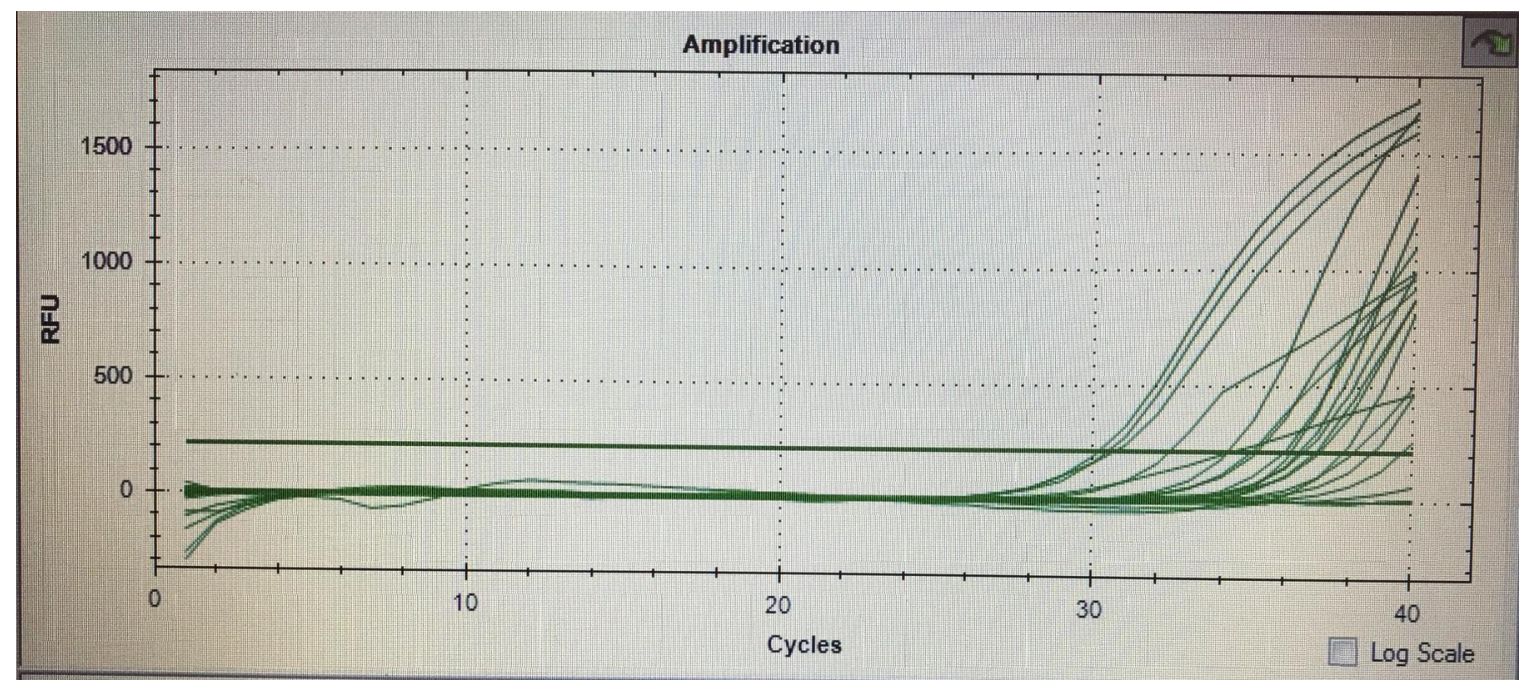

Fig. 4. qRTPCR analysis revealed that unknown goat milk samples obtained from a herd from the American Institute for Goat Research did not have any presence of bacteria, thus The the herd under study was Mastitis free.

* Corresponding author at: American Institute for Goat Research at Langston University, Langston, OK 73034, US. E-mail address: ytilahun@langston.edu (Y. Tilahun) 


\section{Discussion}

Our study detected zero markers for the presence of Mastitis causing bacteria in the selected herd. This method of detection is advantageous and will prevent the spread of Mastitis early if testing is conducted at regular times. The methods described can be utilized for regular disease diagnostics procedures on a number of infected, suspected samples. This method of detection can be used to prevent harmful, invasive diseases from crossing into areas where healthy livestock and/or people reside.

\section{Conflict of interest statement}

The authors have declared that no conflict of interest exists.

\section{Acknowledgements}

Support for this project was made by USDA-AFRI-NIFA. Procedures were completed in the School of Agriculture and Applied Science at Langston University.

Acknowledgements go to the following for support during this study: Mrs. Eva Vasquez, Ms. Ashley Newton, Mr. Magnus Scott.

\section{References}

Akam, D.N., F.H. Dodd, and A.J. Quick. 1989. Milking, milk production and udder health. Food and Agriculture Organizations of the United Nations, FAO Animal Production and Health Paper 78. ISBN 92-5-102661-0

Hurley, W. L. 2009. Mastitis Treatment and Control. In Mastitis: http://ansci.illinois.edu/static/ansc438/index.html. Univerity of illinois UrbanaChampaign.

Petersson-Wolfe, C. S. , I.K. Mullarky, and G.M. Jones. 2010. Staphylococcus aureus Mastitis: Cause, Detection, and Control. VCE Publications, 404:229.

Zestempowska, E. J. Grajewski, and M. Twaruzek. 2016. Food-Borne Pathogens and Contaminants in Raw Milk- a Review. Annals of Animal Science, 16(3):623-639. doi: 10.1515/aoas-2015-0089

* Corresponding author at: American Institute for Goat Research at Langston University, Langston, OK 73034, US. E-mail address: ytilahun@langston.edu (Y. Tilahun) 